\title{
Relación entre áreas y variables ambientales espaciales con el atropellamiento y cruce de la fauna silvestre en la carretera de la Franja Transversal del Norte, Cobán, Guatemala
}

\author{
Areas and spatial environmental variables related with the trampling and wildlife crossing in \\ the Northern Transversal Road in Coban, Guatemala.
}

Oscar Rojas, Carlos Avendaño

Facultad de Ciencias Químicas y Farmacia, Universidad de San Carlos de Guatemala, Guatemala

*Autor al que se dirige la correspondencia: oscaralbertorojascastillo@hotmail.com

Recibido: 04 de abril 2018 / Revisión: 24 de agosto 2018 / Aceptado: 24 de septiembre 2018

\section{Resumen}

$\mathrm{D}$ entro del Proyecto vial de la Franja Transversal del Norte en Cobán se planea asfaltar $10 \mathrm{~km}$ del trayecto en el Parque Nacional Laguna Lachuá. Esto impactará a la fauna de no considerarse medidas de mitigación del atropellamiento. La investigación evaluó los patrones espaciales del atropellamiento de vertebrados, la frecuencia de cruce de aves y categorizó las secciones de la carretera en dicho trayecto, según variables ambientales (humedales, usos del suelo, cobertura; y ancho, asfaltado y ubicación de la carretera) con el fin de determinar los sitios más eficientes para la aplicación de medidas de mitigación. En el 2016, se realizaron 20 recorridos, donde se georreferenció, fotografió e identificó a los individuos atropellados; tres recorridos en los meses de febrero, marzo y abril, cinco en mayo y seis en junio; 12 recorridos fueron realizados adicionalmente cuantificando el cruce de aves para determinar que sitios podrían ser futuramente impactados. Finalmente, para un análisis más completo, el trayecto de la carretera bajo estudio fue dividido en 19 secciones (T1-T19) de $550 \mathrm{~m}$ cada uno. Fueron empleadas: estadística descriptiva, análisis de componentes principales (ACP), correspondencia canónica (CCA) y análisis de correspondencia (AC), para relacionar variables ambientales con atropellamiento y frecuencia de cruce. La mayoría de atropellamientos $(80 \%)$ ocurrieron en zonas asfaltadas, los anfibios fueron los más atropellados (125 individuos, $75 \%$ ), seguido por reptiles 21 (12\%), mamíferos 11 (7\%) y aves 10 (6\%). La mayor frecuencia de cruce ocurrió en zonas con humedales y alta cobertura boscosa (T1, T3, T6, T7 y T10) siendo estas secciones prioritarias para mitigación.

Palabras claves: Patrones espaciales, frecuencia de cruce, sitios de reducción de atropellamiento, cruce de aves, Parque Nacional Laguna Lachuá.

\begin{abstract}
$\mathrm{T}$ The Franja Transversal del Norte road project in the Coban Province plans to pave a $10 \mathrm{~km}$ long dirt road inside the Laguna Lachua National Park. This will impact the fauna if no mitigation measure for wildlife-vehicle collision is considered. The research evaluated the spatial patterns of the vertebrate's roadkills, the bird's road-crossing frequency, and categorized the road section based on environmental variables (wetlands, land use, coverture; and road's width, location, and paved or not paved) to determine the most efficient sites for the application of mitigation measures. In 2016 , 20 treks were made to detect, geo-reference, photograph and identify road killed individuals, three treks in the months of February, March, and April, five in May and six in June; 12 additional treks were made to quantify birds' road-crossing to determine the sites that could be impacted in the future. Finally, for a further analysis, the road section under study was divided into 19 segments (T1-T19), $550 \mathrm{~m}$ long each. A combination of descriptive statistics, principal component analysis (PCA), canonical correspondence analysis (CCA) and correspondence analysis (CA) were employed to relate the spatial environmental variables with roadkill and road-crossing frequency. Eighty percent of the roadkill's occurred in the paved zones, the amphibians were the most affected (125 carcasses, $75 \%$ ), followed by reptiles 21 (12\%), mammals $11(7 \%)$ and birds $10(6 \%)$. The highest road-crossing frequencies were related to wetlands and high forest cover (T1, T3, T6, T7, T10) being priority areas for the application of mitigation measures.
\end{abstract}

Keywords: Spatial patterns, vertebrate road-crossing, wildlife vehicle collision, speed reduction measures, Laguna Lachua National Park. 


\section{Introducción}

Los proyectos viales se consideran de beneficio social y económico por su contribución al desarrollo humano y el mejoramiento de la calidad de vida de los habitantes (Arroyave et al., 2006). Sin embargo, en numerosos estudios se ha observado que el establecimiento de los mismos produce efectos negativos sobre el ambiente, como la fragmentación de ecosistemas, dispersión de especies exóticas, alteración del ciclo hidrológico, modificación micro climática, contaminación auditiva, contaminación física del suelo y agua, y un impacto directo a la flora nativa y principalmente a la fauna (Andrews \& Gibbons, 2008; Dyer, O’Neill, Wasel, $\&$ Boutin, 2002).

La mortalidad de animales por atropellamiento es uno de los efectos negativos más importantes (Trombulak \& Frissell, 2000). Se estima que más de 30 millones de vertebrados son atropellados cada año en España (López, 2004), entre 89 y 340 millones de aves en los Estados Unidos (Loss, Will, \& Marra, 2014), 200,000 ciervos en Alemania (Hothorn, Brandl, \& Müller, 2012) y 5 millones de anfíbios y réptiles en Australia (Hels $\&$ Buchwald, 2001). Dentro de áreas protegidas la cantidad de atropellamientos es aún mayor, en solamente $76 \mathrm{~km}$ de la carretera del Parque Nacional Saguaro en Arizona se estima que más de 29 mil vertebrados son atropellados anualmente (Gerow, Kline, Swann, \& Pokorny, 2010). Estudios de largo plazo estiman que más de 625 serpientes y más de 1,700 sapos son atropellados anualmente en cada $\mathrm{km}$ de carretera cercana a humedales, presentando estas las tasas más altas de atropellamiento de fauna (Ashley \& Robinson, 1996; Forman \& Alexander, 1998).

Por todo lo anterior, el Proyecto vial de la Franja Transversal del Norte (FTN), especialmente la sección ubicada en la Ecorregión Lachuá (EL), debe considerar con seriedad sus repercusiones, ya que esta se caracteriza por una selva tropical lluviosa verdadera, reconocida por su alta biodiversidad faunística, y una serie de cuerpos hídricos que han sido considerados por la convención RAMSAR de importancia nacional e internacional para la conservación de los humedales (Escuela de Biología, 2004). La FTN atraviesa 372 km de esta ecorregión (Rogers, 2013; Solano, 2012). De estos, $10 \mathrm{~km}$ serán pavimentados dentro del Parque Nacional Laguna Lachuá (PNLL) cuya importancia radica en su biodiversidad, ya que resguarda al menos a 130 especies de mamíferos, 30 de reptiles, 40 de anfibios, 177 de aves, incluyendo 29 especies migratorias (Avendaño, 2001; Instituto Nacional de Bosques [INAB], 2003); además el PNLL es el único remanente de bosque tropical verdaderamente lluvioso que se encuentra actualmente protegido en el área, lo que lo hace vital para la conservación de estas especies (Escuela de Biología, 2004).

$\mathrm{Al}$ encontrarse la FTN en contacto con esta importante reserva de vida silvestre, se supone que los impactos del proyecto vial hacia la fauna serán muy altos. Por lo tanto, resulta importante llevar a cabo una medición de los puntos preferenciales de paso de la fauna en la carretera, y complementario a esto, una evaluación de los patrones espaciales de atropellamiento de la misma para priorizar localidades de mitigación del impacto (Teixeira et al., 2013).

La presente investigación tuvo como fin evaluar los patrones espaciales del atropellamiento de vertebrados en los meses de febrero a junio, la frecuencia de cruce de la fauna (aves) del PNLL y caracterizar la carretera tomando en cuenta variables ambientales espaciales (cuerpos hídricos, parche anexo a carretera, traslape de copas (dosel), porcentaje de cobertura boscosa, ancho y tipo de carretera y posición espacial), para así determinar los sitios útiles para la implementación de medidas de reducción del atropellamiento en la misma.

\section{Materiales y métodos}

\section{Área de estudio}

La EL, fisiográficamente pertenece al Cinturón Plegado del Lacandón, es una región kárstica con clima cálido $\left(21-28{ }^{\circ} \mathrm{C}\right)$ y húmedo $(90-95 \%)$ característico de la selva tropical lluviosa verdadera (Ministerio de Agricultura, Ganadería y Alimentación [MAGA] \& Centro Agronómico Tropical de Investigación y Enseñanza [CATIE], 2001). Es habitada por 55 comunidades indígenas de la etnia q'eqchi'. Debido a un historial de ocupación territorial desordenado, debilidades institucionales y de desarrollo social, además de diversas amenazas, entre ellas, talas ilícitas, pérdida de cobertura forestal, expansión de cultivos, proyectos petroleros, entre otros, la riqueza natural de la Ecorregión se encuentra amenazada (Avendaño et al., 2005; INAB, 2003); siendo el PNLL el único remanente de bosque natural protegido en la FTN.

El PNLL es un área de importancia en términos de la biodiversidad del país, posee una extensión de 14,500 ha en la que se encuentran diversos ecosistemas, 
acuáticos y terrestres (planos y de montaña). Dentro del parque se encuentran al menos 130 especies de mamíferos, equivalente a un $50 \%$ de las especies reportadas para el país, y entre un $15-20 \%$ de los reptiles y anfibios (30-40 especies, entre ellas la rana de árbol Hyla ebraccata y la culebra de pantano Tretanorhinus nigroluteus,). El PNLL alberga especies emblemáticas de mamíferos como lo son: el jaguar (Panthera onca), el venado cola blanca (Odocoileus virginianus), el tepezcuintle (Cuniculus paca) y el coche de monte (Pecari tajacu); así como especies de distribución restringida como el cocodrilo moreleti (Crocodilus moreletti) y el mono aullador (Alouatta pigra) (INAB, 2003). Además, es importante para la avifauna neotropical; según estudios por Avendaño (2001), reportan 177 especies, incluyendo 13 de las 17 especies endémicas regionales y 29 especies migratorias, lo que equivale aproximadamente al $44 \%$ del total del país (Avendaño, 2001).

\section{Recolección de datos:}

El procedimiento de este estudio consistió en tres fases. La primera fue la observación de los vertebrados atropellados, la segunda fue la determinación de la frecuencia de cruce de aves en la carretera y la tercera fue la caracterización de los tramos de la carretera en función de variables espaciales, finalmente se realizó un análisis de estas con los fenómenos de atropellamiento $\mathrm{y}$ de paso de aves.

Detección del atropellamiento de vertebrados. En cinco meses se llevaron a cabo 20 recorridos en bicicleta a lo largo de la carretera del límite norte del PNLL a una velocidad promedio de $20 \mathrm{~km} / \mathrm{h}$. Cada recorrido fue de $10 \mathrm{~km}$, y en cada uno se observó, identificó a nivel de especie (Identificación de la herpetofauna Köhler, 2008; y de la ornitofauna empleando van Perlo, 2006), anotó y georreferenció los vertebrados atropellados. Los recorridos se llevaron a cabo por conveniencia (según el tiempo de estadía del investigador en el área). En febrero, marzo y abril se realizaron tres recorridos. En mayo se realizaron cinco recorridos y en junio seis; abarcando meses de temporada seca (febrero, marzo y abril) y meses de temporada lluviosa (mayo y junio).

Caracterización de los tramos de la carretera en función de variables ambientales espaciales. El total de la FTN que limita la sección norte del PNLL se dividió en 19 unidades o tramos de 550 m cada uno (denominados del T1 al T19, como se muestra en la fi- gura $1 \mathrm{~A}, 1 \mathrm{~B}$ y $1 \mathrm{C})$, los primeros 13 fueron de terracería y los últimos seis de asfalto. Las unidades se caracterizaron mediante la observación y el análisis de imágenes satelitales en el programa Google Earth $($, en función de las variables ambientales espaciales: presencia de cuerpos de agua (detectando cuerpos de agua anexos a la carretera observables en las imágenes satelitales y/o recorridos), identificación de parche anexo a carretera (clasificados en función de las categorías: bosque, jardín/vivienda cultivo, pastizal, palma africana), porcentaje de traslape de copas sobre la carretera (empleando una cuadrícula $0.5 \times 0.5 \mathrm{~cm}$ sobre la carretera de la imagen satelital de Google Earth@ escala 1:2,255 (Google, 2015), se calculó el porcentaje de cuadros con cobertura), porcentaje de cobertura de parches anexos (100 m al norte y $100 \mathrm{~m}$ al sur fueron analizados con la misma cuadrícula e imagen que la variable anterior), sustrato del camino (asfalto o terracería), ancho de carretera (medida directamente en los puntos de observación de cruce de la fauna) y posición espacial (se obtuvo la latitud, longitud y altitud de Google Earth $\odot$ mediante un GPS Garming $($ ) (Tabla 2).

\section{Medición de la frecuencia de cruce de aves}

Cada una de las 19 unidades (T1 al T19) se representó por una subunidad de $200 \mathrm{~m}$ ubicada dentro de las mismas, esta es la distancia máxima continua que permite una observación ininterrumpida para detectar eventos de paso o cruce de animales en la carretera del norte del PNLL (distancia calibrada en premuestreo, mediante la realización de observaciones en diferentes puntos de la carretera con el fin de evitar sesgo causado por eventos de cruce no observados consecuencia de obstrucciones como ramas o curvas en la carretera). Cada subunidad fue observada por un periodo de $5 \mathrm{~min}$ en cada recorrido de visualización de paso (un total de 12 recorridos por unidad, seis de los cuales llevados a cabo en mayo y los seis restantes en junio). Cada unidad se observó durante el mismo tiempo en los distintos intervalos temporales planteados (6:00-7:30, 7:30-9:00, 9:00-10:30, 10:30-12:00), con el fin de reducir el sesgo que puede darse en función de la variación de la actividad de los vertebrados a lo largo del día.

\section{Análisis de datos}

Las frecuencias de cruce de aves y del atropellamiento fueron analizadas empleando estadística 


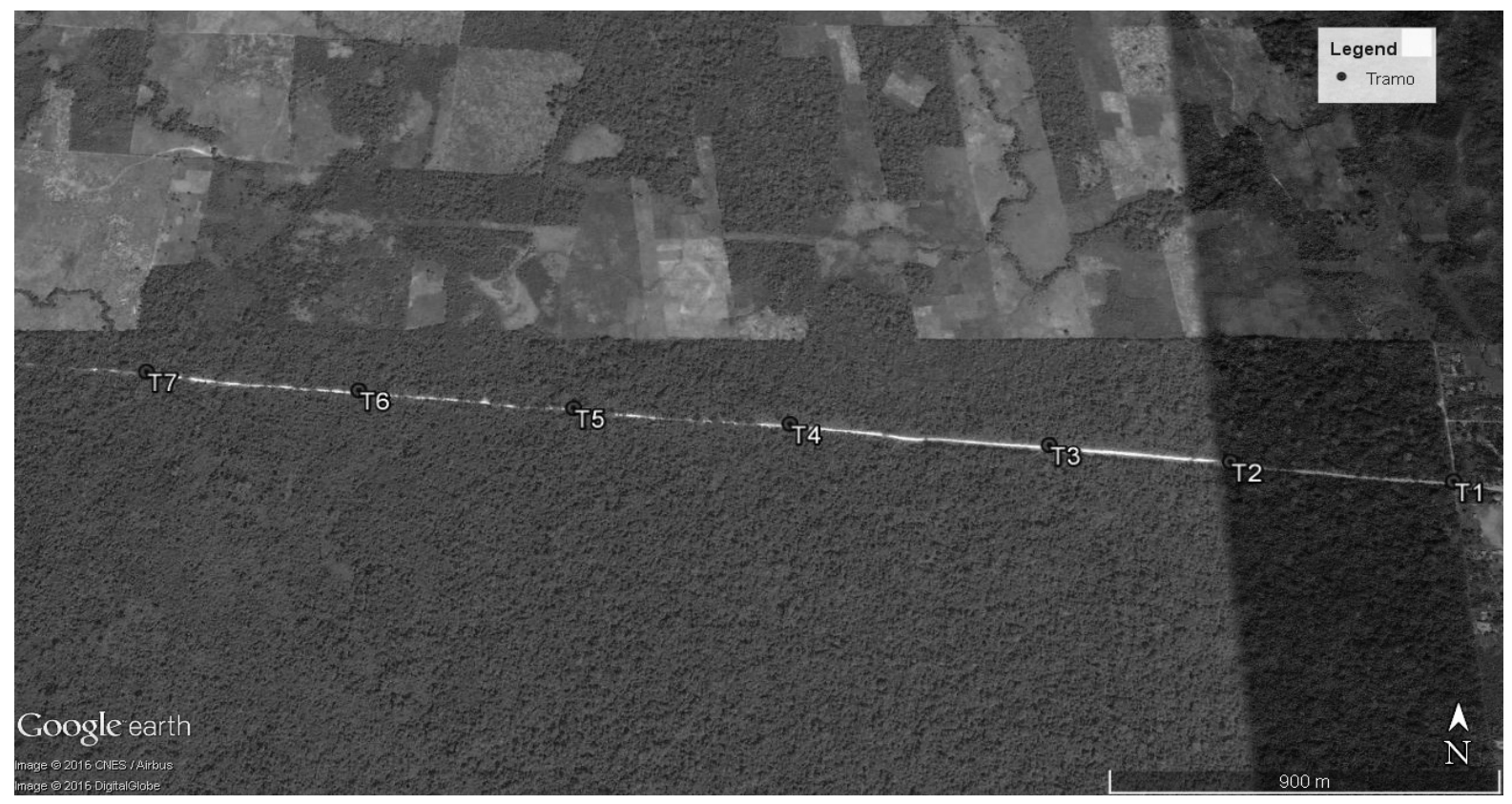

Figura 1A. Tramos T1-T7 del Proyecto de la FTN, ubicado en el norte del Parque Nacional Laguna Lachuá (Google Inc, 2015).

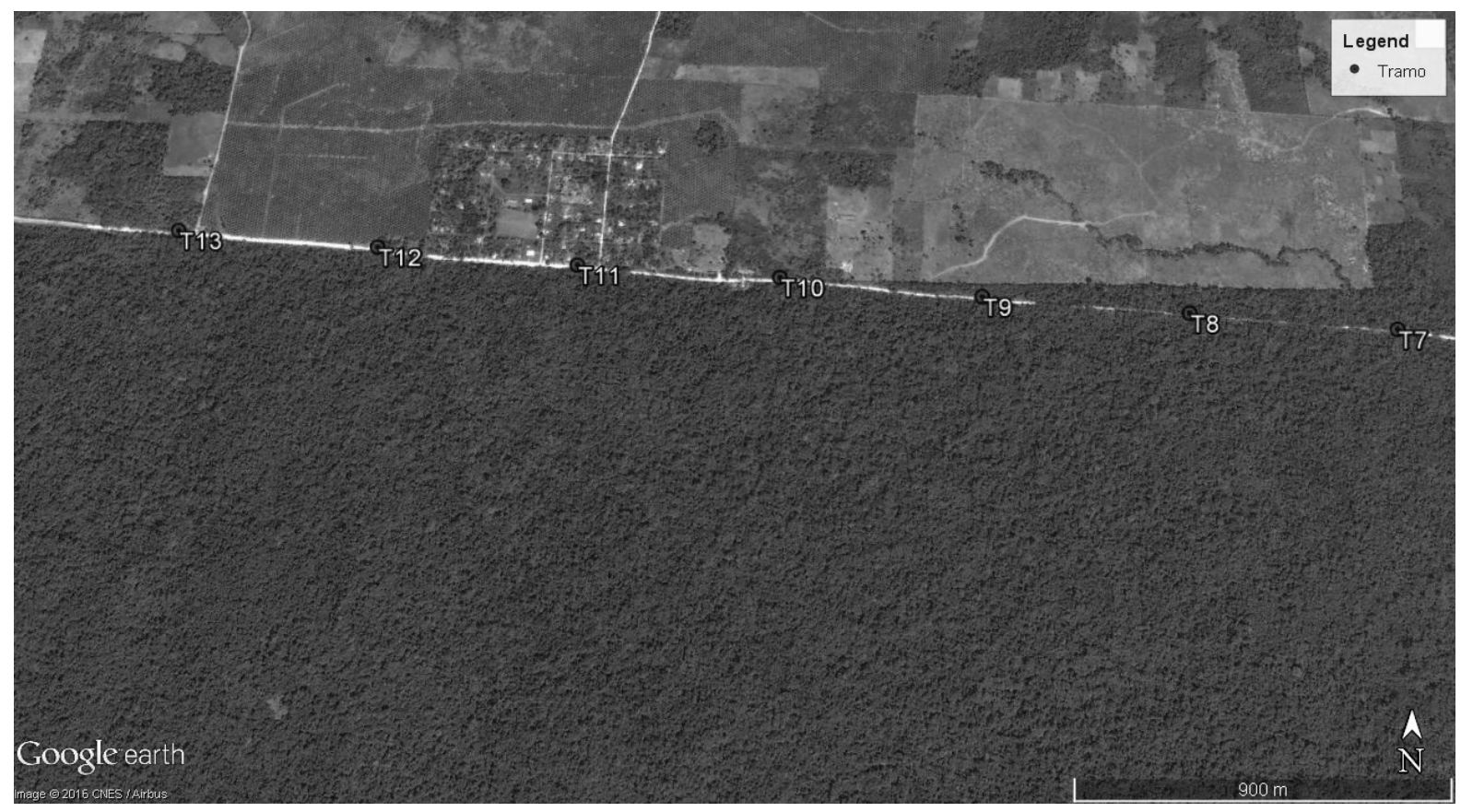

Figura 1B. Tramos T8-T13 del Proyecto de la FTN, ubicado en el norte del Parque Nacional Laguna Lachuá (Google Inc, 2015). 


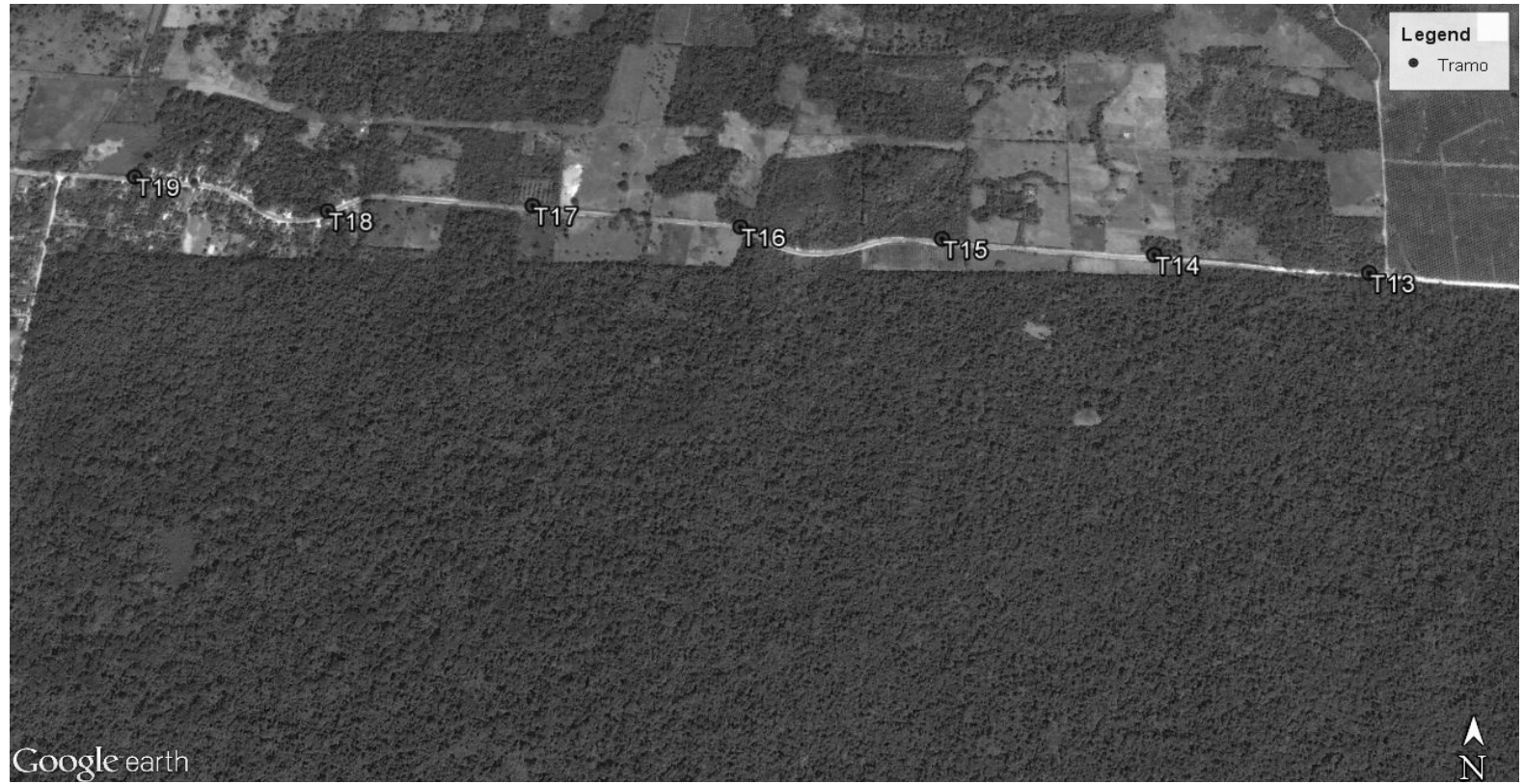

Figura 1C. Tramos T13-T19 del Proyecto de la FTN, ubicado en el norte del Parque Nacional Laguna Lachuá (Google Inc, 2015).

Cada uno de los 19 tramos T1, T2, T3, T4, T5, T6, T7 (Figura 1A), T8, T9, T10, T11, T12 (Figura 1B), T13, T14, T15, T16, T17, T18, T19 (Figura 1C) mide 550 metros y fue caracterizado en función de variables ambientales espaciales, estos tramos representan los ejes $\mathrm{x}$ de las figuras 2 y 3 .

descriptiva, utilizando diagramas de barras (Cooper \& Shore, 2010). Además, se utilizaron los análisis de componentes principales (PCA), análisis de correspondencia canónica (CCA) y análisis de correlación (CA) en Past 3.03 (Hammer, Harper, \& Ryan, 2001), con el fin de observar las variables ambientales espaciales relacionadas a la frecuencia de cruce (PCA y CCA) y al atropellamiento (CA), para entender estos fenómenos de una forma más detallada (Legendre \& Legendre, 1998; McGarigal, Stafford, \& Cushman, 2000).

\section{Resultados}

Los sitios asfaltados presentaron un mayor número de animales atropellados en contraste con los sitios de terracería. En los $3.24 \mathrm{~km}$ de asfalto del área de estudio (tramos T14-T19), el número de vertebrados fue de 132 (103 anfibios, 5 mamíferos, 15 réptiles y 9 aves), considerablemente mayor al observado en los $6.86 \mathrm{~km}$ de terracería, donde el valor fue de 35 (22 anfibios, 6 mamíferos, 6 réptiles y 1 ave) (Figura 2). Los tramos T16 y T17 presentaron el mayor atropellamiento. Los anfibios fueron los más atropellados, seguido por los reptiles, mientras las aves fueron las menos atropelladas. Los tramos T6, T9, T10 y T13 presentaron la mayor cantidad de animales atropellados en lo que respecta a la terracería de la carretera (Figura 2).

El taxón más frecuentemente atropellado fue el de anfibios con 125 (75\%) individuos, seguido por réptiles con 21 (12\%) individuos, mamíferos con 11 (7\%) individuos y por último el de aves con $10(6 \%)$ individuos siendo un total de 167 animales silvestres atropellados (Figuras 2 y 4).

Once mamíferos fueron atropellados. El tacuazín (Didelphis marsupialis) fue la especie con el mayor número (10) (Tabla 1). Diez aves fueron atropelladas, la familia Trochilidae (Phaethornis superciliosus y Amazilia tzacatl) fue la más afectada con dos atropellamientos. Ciento veinticinco anfibios fueron atropellados y debido al deterioro de los organismos por el sol, lluvia, etc. no todos se lograron identificar a nivel de especie; 85 individuos se identificaron hasta familia, 65 hasta género y 57 hasta especie. Rhinella horribilis fue la más afectada de este grupo con 36 atropellamientos, seguida por Incilius valliceps con 10 (Tabla 1). Veinte reptiles fueron atropellados, $15 \mathrm{del}$ grupo serpentes (serpientes), 


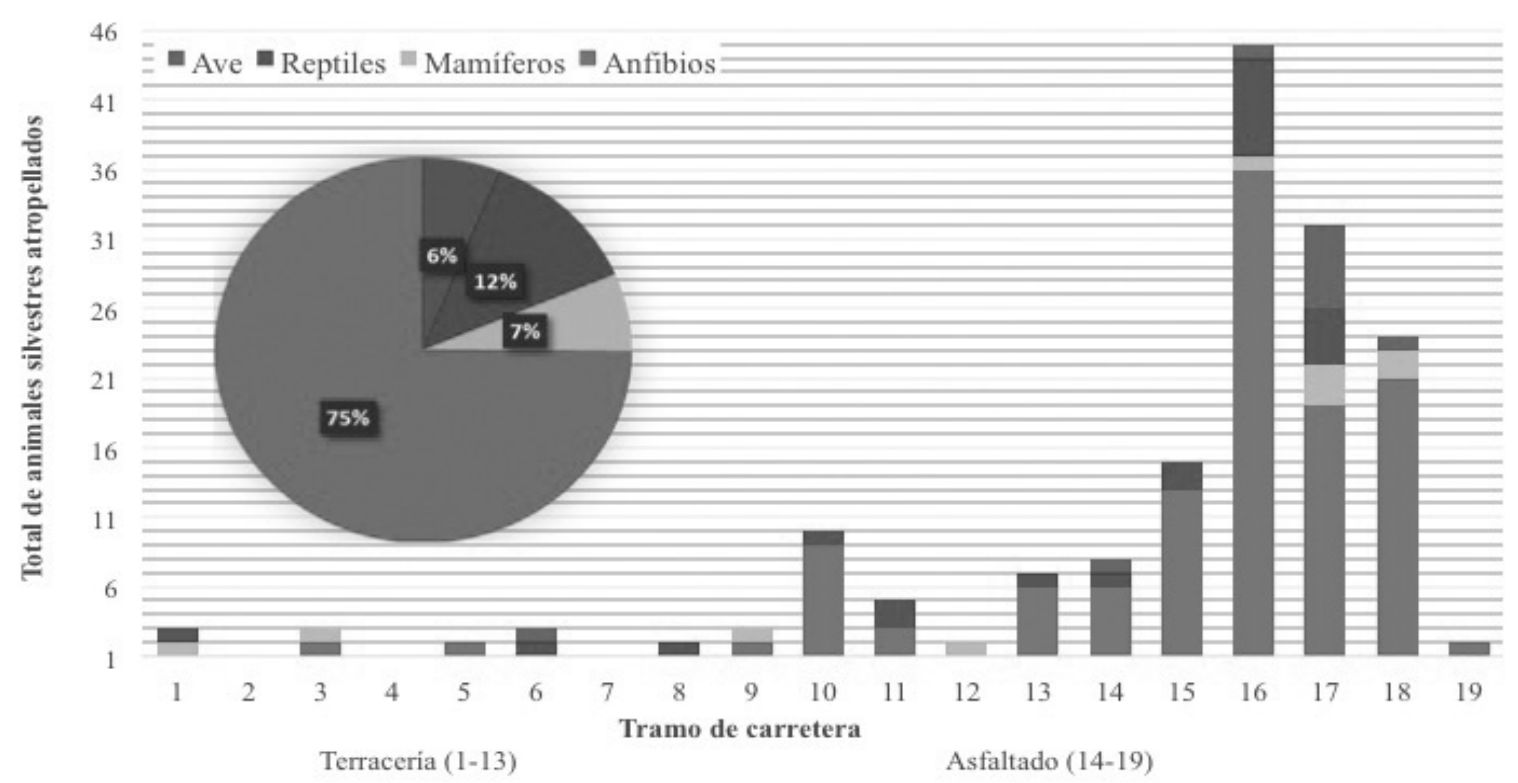

Figura 2. Proporción de vertebrados atropellados según su taxón y total de atropellamientos detectados por tramo.

dos del grupo testudines (tortugas) y dos del grupo lacertilla (lagartijas), siendo Ninia sebae y Rhadinaea decorata los más atropellados (Tabla 1 ).

Los tramos T6 y T7 de la sección de terracería, asociados a ríos, presentaron los mayores valores de paso de aves (Figura 3). El tramo T12, asociado a un parche de cultivo de palma africana (Elaeis guineensis) considerada una especie exótica en el país, fue el sitio con el menor paso de aves (Figura 3). En la sección asfaltada los tramos T14 (pastizal) y T17 (pequeño bosque), presentaron los valores mayores de frecuencia de cruce de aves.

Los sitios 1-9 se caracterizaron por no presentar asfalto, un ancho de carretera menor a $5 \mathrm{~m}$, cobertura forestal mayor a $60 \%$, los menores valores de longitud (X) y latitud (Y) encontrándose más al sur y este que el resto de los tramos; además presentaron un parche anexo de bosque. Los tramos 10-12, fueron de terracería, cobertura forestal menor a $60 \%$, colindaron al sur con parches de bosque y al norte con parches de casas y jardines (tramo 10-11) y palma africana (tramo 12). El tramo 13 fue muy similar a los tramos 1-9, sin embargo, además de bosque al norte colindó con un parche de pastizal $(50 \%)$ y el ancho de la carretera era mayor a $5 \mathrm{~m}$. Los tramos 3, 6, 7, 9, 10, 12 y 15 se encuentran anexos a un humedal (río 6, 7 y 10; laguna 3, 9, 12 y 15); los tramos $4,5,7$ y 8 presentan un traslape de copas alto (mayor al $50 \%$ ). Los tramos $14-17$ presentaron asfalto, alta cobertura forestal (mayor a $60 \%$ ), bajo traslape de copas y parches anexos de cultivos (tramo 14 y 15), pastizales (13-14, 16-17), casas (18-19) y bosque $(13,15$ y 17).

Para el CA y PCA se utilizaron las variables ambientales espaciales observadas en la Tabla 2 con respecto al atropellamiento (CA) o al paso de aves (PCA). En el CA las variables de posición (latitud y longitud) presentaron correlación positiva significativa con la mortalidad de anfibios $(p<.05)$, y con la mortalidad de aves $(p<.05)$ lo que significa que un mayor número de atropellamientos ocurrió en el norte y oeste. El ancho de la carretera también presentó correlación significativa con la variable de mortalidad de anfibios $(p<.05)$. Con relación al PCA, el eje 1 explica casi el $50 \%$ de la varianza total. Las variables presencia de humedales y porcentaje de cobertura se relacionaron positivamente $(p<.05)$ con la variable de frecuencia de cruce, mientras que la variable altitudinal se relacionó de forma inversa con la variable de frecuencia de cruce (Figura 4).

\section{Discusión}


Tabla 1

Lista de vertebrados y sus abundancias en eventos de atropellamiento en la carretera de la FTN de la EL (febrero-junio, 2015)

\begin{tabular}{|c|c|c|c|c|c|}
\hline Grupo & $\begin{array}{c}\text { No. de } \\
\text { Individuos }\end{array}$ & Grupo & $\begin{array}{c}\text { No. de } \\
\text { Individuos }\end{array}$ & Grupo & $\begin{array}{c}\text { No. de } \\
\text { Individuos }\end{array}$ \\
\hline Mamíferos & 11 & Reptiles & 20 & Anfibios & 125 \\
\hline $\begin{array}{l}\text { Didelphis } \\
\text { marsupialis }\end{array}$ & 10 & Reptiles (Lacertilia) & 3 & No identificable & 40 \\
\hline \multirow[t]{5}{*}{$\begin{array}{l}\text { Conepatus } \\
\text { semistriatus }\end{array}$} & 1 & Corytophanes cristatus & 1 & $\begin{array}{l}\text { Smilisca } \\
\text { baudinii }\end{array}$ & 1 \\
\hline & & Basiliscus pilatus & 1 & Agalychnis calidryas & 1 \\
\hline & & Coleonyx elegans & 1 & $\begin{array}{l}\text { Rhinophrynus } \\
\text { dorsalis }\end{array}$ & 6 \\
\hline & & Reptiles (Testudines) & 2 & Hylidae & 2 \\
\hline & & Dermatemys mawii & 1 & Lithobates vaillanti & 2 \\
\hline Aves & 10 & Trachemys scripta elegans & 1 & $\begin{array}{l}\text { Lithobates } \\
\text { brownorum }\end{array}$ & 1 \\
\hline No identificable & 6 & Reptiles (Serpentes) & 15 & Lithobates Sp. & 8 \\
\hline Amazilia tzacatl & 1 & No identificable & 1 & Rhinella horribilis & 36 \\
\hline $\begin{array}{l}\text { Platyrinchus } \\
\text { cancrominus }\end{array}$ & 1 & Micrurus nigrocinctus & 1 & $\begin{array}{l}\text { Incilius } \\
\text { valliceps }\end{array}$ & 10 \\
\hline $\begin{array}{l}\text { Phaethornis } \\
\text { superciliosus }\end{array}$ & 1 & $\begin{array}{l}\text { Scaphiodontophis } \\
\text { annulatus }\end{array}$ & 1 & Buffonidae & 18 \\
\hline \multirow[t]{7}{*}{ Habia fuscicauda } & 1 & Porthidium nasutum & 1 & & \\
\hline & & Imantodes cenchoa & 1 & & \\
\hline & & Ninia diademata & 1 & & \\
\hline & & Ninia sebae & 3 & & \\
\hline & & Leptodeira septentrionalis & 2 & & \\
\hline & & Rhadinaea decorate & 3 & & \\
\hline & & Boa constrictor & 1 & $\begin{array}{l}\text { Total }=167 \\
\text { individuos }\end{array}$ & \\
\hline
\end{tabular}




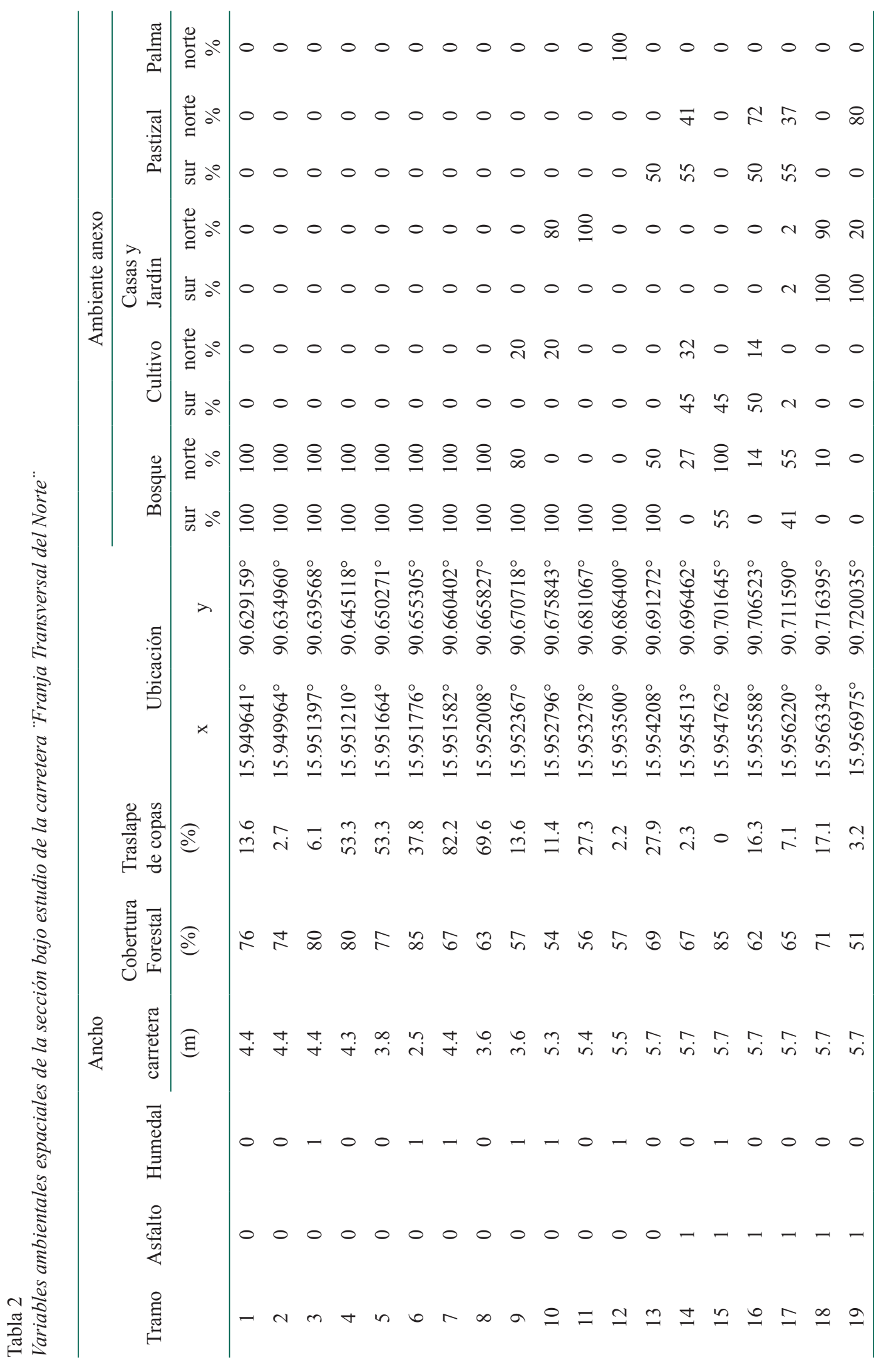




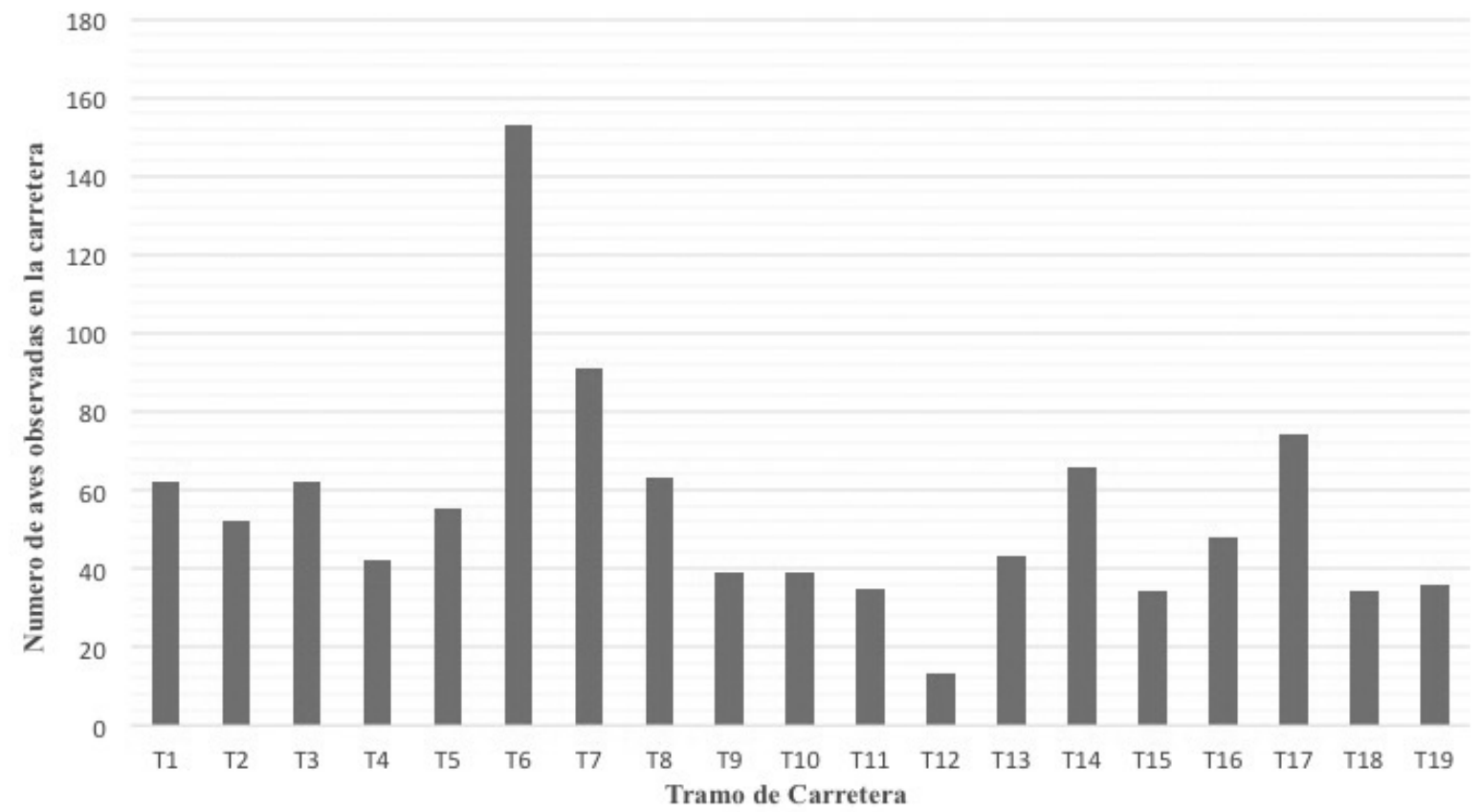

Figura 3. Frecuencia de cruce de aves por la carretera.

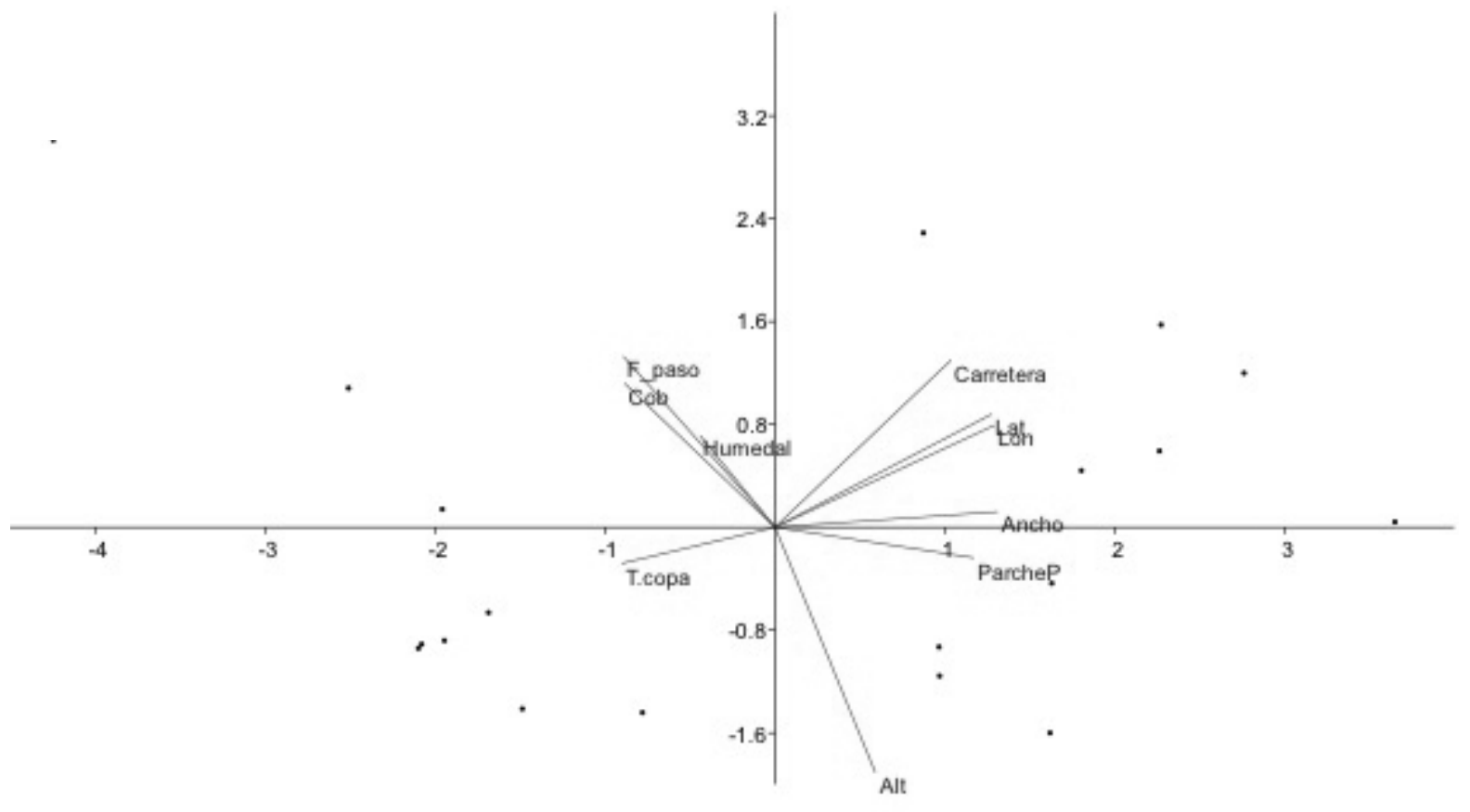

Component 1

Figura 4. Análisis de Componentes Principales (PCA) de las variables ambientales espaciales y de frecuencia de cruce de aves Abreviaturas: F paso: Frecuencia de paso; Lat: Latitud; Lat: Longitud; ParcheP: Parche principal; Cob: Cobertura; Alt: Altitud; Humedales: Humedales cercanos; T.copa: Traslape de copa en carretera. 
El área asfaltada presentó valores de atropellamiento notablemente mayores a los observados en el área de terracería (Figura 2) lo cual explica la correlación positiva $(p<.05)$ entre la posición y el atropellamiento. Los sitios asfaltados se localizaron en las mayores latitudes y longitudes y estos presentaron mayor atropellamiento. El efecto del asfalto ha sido reportado en numerosos estudios y es asociado a la velocidad vehicular que permite, al proporcionar una superficie plana sin irregularidades los vehículos pueden movilizarse con mayores velocidades que en áreas de terracería, donde las irregularidades son numerosas (Ament, Clavenger, Yu, \& Hardy, 2008; Arroyave et al., 2006). Sin embargo, la velocidad vehicular no fue medida en el presente estudio. Esta diferencia en cuanto al número de atropellamientos en función del asfaltado evidencia un probable aumento en la mortalidad de la fauna silvestre cuando el asfaltado de la carretera FTN finalice, reflejando la importancia de la implementación de reductores de velocidad y/o medidas de mitigación del atropellamiento de fauna en la carretera.

De manera similar a lo establecido en estudios previos (Ashley \& Robinson, 1996; Glista, DeVault, \& De Woody, 2008; Rodríguez, 2015), la herpetofauna (anfibios y réptiles) presentó los mayores valores de atropellamiento de todos los taxa en el presente estudio (Figura 2 y Tabla 1). Este fenómeno se atribuye a la naturaleza de este grupo. Las carreteras suelen ser un elemento atractivo para la herpetofauna, debido a su naturaleza ectotérmica, y requieren regular su temperatura corporal mediante la absorción de calor del medio (Ashley \& Robinson, 1996). Las carreteras pavimentadas absorben grandes cantidades de calor durante el día, debido a esto en la noche actúan como fuentes de calor, lo que es aprovechado por la herpetofauna para la regulación de su temperatura corporal (Ashley \& Robinson, 1996; Arroyave et al., 2006). Lo anterior hace que esta fauna se encuentre altamente expuesta al atropellamiento.

Los anfibios, al igual que en los estudios de Andrews y Gibbons (2008) y Mazerolle (2004), fueron los más atropellados (Figura 2 y Tabla 1). Este fenómeno es explicado en función del comportamiento, ya que al movilizarse los anfibios en la carretera realizan paradas periódicas aumentando su tiempo en las mismas y con esto su probabilidad de ser atropellados (Andrews \& Gibbons, 2008; Mazerolle, 2004). El sapo común ( $R$. horribilis) fue el anfibio con los mayores valores de atropellamiento (Tabla 1) lo cual ha sido observado en estudios previos y ha sido asociado a su alta abundancia en Brasil (Junior, Lima, Santos, \& Arid, 2012) y Costa Rica (Rodríguez, 2015). El atropellamiento de anfibios tiene una relación directa con el ancho de la carretera (Figura 4), en primer lugar, porque la zona asfaltada es la más ancha y en segundo lugar porque tienen más probabilidad de ser atropellados al cruzar caminos anchos, debido a su lenta y pausada movilidad (Mazerolle, 2004). Un hallazgo interesante en este taxón fue la observación de 10 individuos atropellados pertenecientes a la especie Rhinophrynus dorsalis, siendo la cuarta especie más atropellada en este grupo (Tabla 1). Este evento tiene importancia ecológica, ya que esta especie permanece enterrada durante casi todo el año, saliendo de sus agujeros únicamente en la época de reproducción que coincide con la época lluviosa (Sandoval, Barrantes, Ocampo, \& Sánchez-Quirós, 2015) siendo esta la época en que se observó el $100 \%$ de los atropellamientos de la especie, pudiendo afectar a la dinámica de la población y con esto su rol ecológico en un futuro. Además, $R$. dorsalis es el único sobreviviente de un linaje independiente y antiguo derivado de los ascafidos durante el Cretácico, lo que la hace importante a nivel evolutivo y por consecuente importante en la conservación de la diversidad genética (Foster \& McDiarmid, 1983; Frost, 2013; Maxson \& Daugherty, 1980).

En los reptiles las especies con los valores más altos de atropellamiento fueron, $R$. decorata y $N$. sebae (Tabla 1), dos serpientes bastante comunes en Mesoamérica, las cuales también han sido reportadas como las más atropelladas en investigaciones previas (Rodríguez, 2015). A pesar de que las aves fueron los únicos vertebrados observados cruzando la carretera (Figura 3), estos presentaron las menores frecuencias de atropellamiento, probablemente por su alta agilidad y velocidad de cruce asociada al vuelo (Baskaran \& Boominathan, 2010); además no se observó dominancia de atropellamiento en una especie si no en los integrantes de la familia Trochilidae (colibrís), la cual presentó una ligera dominancia de atropellamiento con dos integrantes (P. superciliosus y A. tzacatl) (Tabla 1), esto probablemente porque los colibrís se alimentan de néctar y existe un gran número de flores en las orillas del parque, actuando estas como atrayentes. La frecuencia de cruce de aves se correlaciona directamente con las variables de cobertura forestal y con la presencia de humedales (Figura 4). Arroyave y colaboradores (2006), explican el efecto de la primera variable con el alto número de individuos y especies que habitan los bosques. Glista y 
colaboradores (2008) por otra parte, relacionan el efecto de la segunda variable con el efecto de corredores biológicos que presentan los ríos y cuerpos de agua.

El mamífero más atropellado fue el tacuazín (D. marsupialis), lo cual concuerda con la bibliografía en el caso de Brasil (Gumier-Costa \& Sperber, 2009; Junior et al., 2012) y Costa Rica (Rodríguez, 2015). Este hecho es explicado por su hábito de oportunismo (basureros) y depredador de insectos, pequeños reptiles y anfibios fácil de atrapar en lugares abiertos. Además, esta especie aprovecha las carreteras para llevar a cabo una movilización ininterrumpida, ya que las carreteras, al no contar con vegetación brindan un camino sin obstáculos (Junior et al., 2012). Sin embargo, los datos del atropellamiento de los mamíferos podrían estar sesgados, debido a que según los guarda-recursos del PNLL muchos de estos atropellamientos no dejan evidencia, ya que los habitantes de esta zona tienden a llevarse animales como coches de monte, cabritos, jabalís, armadillos e incluso tacuazines para alimentación después de atropellarlos.

El tramo T6 es un sitio de importancia para el atropellamiento y la frecuencia de cruce (Figura 2 y 3). La alta frecuencia de cruce en este caso (Figura 3) además de estar correlacionado con la presencia de un humedal amplio y un alto nivel de cobertura forestal (Figura 4 y Tabla 2), puede explicarse en función de su complejidad paisajística al contar con bosque alto y mediano, pero también con una zona de gramíneas (familia Poaceae) y un río de gran tamaño. Esta diversidad de microhábitats promueve la presencia de diferentes recursos en lados opuestos de la carretera, lo que propicia el cruce de la fauna por la misma (Andrews \& Gibbons, 2008; Carr \& Fahrig, 2001). A pesar de no correlacionarse el ancho de la carretera con la frecuencia de cruce de manera clara en el estudio (Figura 4), esto si se ha reportado en diversos estudios, al relacionarse las barreras ecológicas estrechas con una disminución de la fragmentación del hábitat y por lo tanto promoviendo la conectividad de las poblaciones de fauna (Arroyave et al., 2006). La alta frecuencia de cruce en el tramo T6 se explica en función del reducido ancho del tramo de la carretera, cerca de $5 \mathrm{~m}$.

A pesar de que en el tramo $\mathrm{T} 6$ de la carretera es de terracería (Tabla 2) y por lo tanto no favorece el tránsito continuo y veloz de los automóviles, si favorece el paso de animales debido principalmente a que un río de gran tamaño atraviesa el tramo manteniendo la conectividad del hábitat ripario (Rodríguez, 2015). Lo anterior explica la presencia de reptiles, anfibios y aves atropellados (Figura 2). El tramo T6, al promover el paso de la fauna silvestre, (Figura 3) y al mostrar evidencia de atropellamiento durante los 5 meses de monitoreo (es uno de los sitios con más fauna atropellada en terracería) (Figura 2). El tramo T6 se considera importante para la implementación de medidas de mitigación del impacto del atropellamiento.

El tramo T7 es también un sitio de importancia para el paso de animales, reportando el valor más abundante después del tramo T6 (Figura 3), ya que a pesar de no contar con tan alta diversidad de microhábitats como el tramo T6 cuenta con un río (Tabla 2) que podría estar actuando como un corredor biológico lo que se ha evidenciado en diversos ríos (Rodríguez, 2015). El T7 presenta alta conectividad entre copas (bosque alto), lo cual favorece el paso de especies que habitan este tipo de bosque (Arroyave et al., 2006). La inexistencia de atropellamiento en este sitio se debe al estado deteriorado de la carretera, a consecuencia de los ríos que atraviesan dicho tramo, arrastrando rocas y humedad que va erosionando el sustrato del tramo. Sin embargo, al terminarse el proceso de asfaltado en este tramo, se predice un aumento considerable del atropellamiento, debido a que presenta un valor alto de frecuencia de paso.

Los Tramos T1, T3 y T10 también presentan importancia en lo que respecta a la propuesta de reducción del atropellamiento. El Tramo T1 además de poseer valores altos de frecuencia de cruce de aves (Figura 3) y presencia de atropellamiento de mamíferos, anfibios y réptiles (Figura 2), también se encuentra vecino a un centro urbano incipiente (Santa Lucía Lachuá). Estas medidas serían funcionales no solo para la protección de la fauna del PNLL, sino también para la protección de las personas en la comunidad Santa Lucía. El tramo 3 presentó una alta frecuencia de cruce (Figura 3) y un valor de atropellamiento relativamente alto (cinco individuos), similar a los observados en el Tramo T1 (Figura 2). Esta similitud sugiere al T3 como un sitio importante en lo que respecta a la implementación de medidas de reducción contra el atropellamiento. El Tramo T10 también se considera importante para la implementación de dichas medidas, ya que presenta un valor de atropellamiento bastante alto, el mayor observado en el sitio de terracería (Figura 2). Además de la importancia individual de cada tramo seleccionado para la implementación de reductores de velocidad, la distribución de los mismos es también estratégica, evitando tramos continuos muy largos que permitan movilización de vehículos a velocidades altas (Arroyave et al., 2006).

Tomando en cuenta los valores de atropellamiento, los Tramos T16, T17 y T18 fueron identificados 
como sitios importantes para la implementación de medidas de reducción de atropellamiento en la zona asfaltada, al ser los que presentaron los valores de atropellamiento más elevados (Figura 2). Los tramos T11 y T19 se consideran importantes, ya que se ubican dentro de comunidades humanas.

El Tramo T12 presentó el menor paso de aves (Figura 3). Esto podría explicarse en función al cultivo de palma africana al que se encuentra anexo (Tabla 2). El monocultivo ha sido asociado en diversas investigaciones científicas, con una reducción de especies de aves, mamíferos, anfibios e insectos (Savilaakso, Petrokofsky, Zrust, \& Guariguata, 2014). Además de la reducción de la riqueza de especies, el monocultivo de palma africana también se ha visto asociado con una reducción en la abundancia de muchos de estos grupos, principalmente en aves y mamíferos (Foster et al., 2011). Esta pérdida de diversidad puede explicarse en función de la simplicidad del paisaje, tanto en la diversidad vegetal como en su arquitectura y fisonomía, lo cual reduce la diversidad de nichos (Foster et al., 2011; Savilaakso et al., 2014). Otra importante razón de la pérdida de diversidad se explica en función del microclima que permite el dosel. El dosel de la palma africana es bajo, abierto y mucho más simple (formado por una sola especie) que el del bosque lluvioso, lo cual se encuentra asociado a condiciones desfavorables de temperatura y humedad para muchos organismos (Foster et al., 2011). Estos cambios presentan dos componentes: Condiciones extremas (alta temperatura y baja humedad), y mayor variabilidad de estos componentes a lo largo del día. Estas condiciones no son favorables en el área anexa al PNLL, ya que pueden incidir en el aislamiento de la fauna que habita en el parque.

De manera general, se recomienda la instalación de señales que prevengan a los conductores sobre el cruce de animales en la carretera; sin embargo, la efectividad de esta medida de mitigación es muy variable (Beckmann, Clevenger, Huijser, \& Hilty, 2010). La construcción de túneles para el paso de animales podría disminuir los impactos del atropellamiento considerablemente (Lesbarrères, Lodé, \& Merilä, 2004), además traería otros beneficios relacionados a la red hídrica al permitir el flujo del agua. Se recomienda, además, el establecimiento de una garita en el límite este y oeste del parque (tramos T1 y T19) acompañada de una serie de reductores de velocidad colocados en los sitios importantes para la implementación de medidas de reducción del atropellamiento (tramos: T3, T6, T7, T10, T11, T16, T17 y T18; priorizando el T6), obligando al conductor a reducir su velocidad y con esto mitigar efectivamente el atropellamiento de la fauna del PNLL y sus alrededores (van der Grift et al., 2013).

\section{Agradecimientos}

Se agradece al estudiante de biología Renato Morales por el apoyo en la identificación de la herpetofauna atropellada, al guarda-recursos Paulino Baleu por el apoyo en la identificación de la ornitofauna, y a Luis Trujillo por el apoyo brindado durante sus prácticas de EPS. También agradecemos a todos los integrantes del Parque Nacional Laguna Lachuá, sin los cuales este estudio no habría sido posible.

\section{Referencias}

Ament, R., Clevenger, A. P., Yu, O., \& Hardy, A. (2008). An assessment of road impacts on wildlife populations in US National Parks. Environmental Management, 42(3), 480. doi:10.1007/s00267008-9112-8.

Andrews, K. M., \& Gibbons, J. W. (2008). Roads as catalysts of urbanization: snakes on roads face differential impacts due to inter-and intraspecific ecological attributes. En J. C. Mitchell, R. E. Jung Brown, \& B. Barthdomenw (Eds.), Urban Herpetology, (pp. 145-153), Salt Lake City, Utah: Society for the study of Amphibians and Reptiles.

Arroyave, M. P., Gómez, C., Gutiérrez, M. E., Múnera, D. P., Zapata, P. A., Vergara, I. C., ... Ramos, K. C. (2006). Impactos de las carreteras sobre la fauna silvestre y sus principales medidas de manejo. Revista EIA, 5(1), 45-57.

Ashley, E. P., \& Robinson, J. T. (1996). Road mortality of amphibians, reptiles and other wildlife on the Long Point Causeway, Lake Erie, Ontario. Canadian Field Naturalist, 110(3), 403-412.

Avendaño, C. (2001). Caracterización de la Avifauna del Parque Nacional Laguna Lachuá, Cobán, Alta Verapaz (Tesis de licenciatura). Universidad de San Carlos de Guatemala, Facultad de Ciencias Químicas y Farmacia, Guatemala.

Avendaño, C.E, Avendaño, C., Carías, A., García, M., Cajas, M. \& De León, K. (2005). Dinámica del uso de la tierra y conservación de los Recursos 
Naturales de la Eco-región Lachua. Consejo Nacional de Ciencia y Tecnología, Escuela de Biología, Universidad de San Carlos de Guatemala. Recuperado de http://glifos.concyt.gob.gt/digital/ fodecyt/fodecyt $\% 202003.20 . p d f$

Baskaran, N., \& Boominathan, D. (2010). Road kill of animals by highway traffic in the tropical forest of Mudumalai Tiger Reserve southern India. Journal of Threatened Taxa, 2(3),753-759. doi: 10.11609/ JoTT.o2101.753-9

Beckmann, J. P., Clevenger, A. P., Huijser, M. P., \& Hilty, J. A. (2010). Safe passages high ways, wildlife, and habitat connectivity, (March 2014), 396. doi:10.2307/23259952.

Carr, L. W., \& Fahrig, L. (2001). Effect of road traffic on two amphibian species of differing vagility. Conservation Biology, 15(4), 1071-1078. doi:10.1046/j.1523-1739.2001.0150041071.x.

Cooper, L. L., \& Shore, F. S. (2010). The effects of data and graph type on concepts and visualizations of variability. Journal of Statistics Education, 18(2), 1-16. doi:10.1080/10691898.2010.11889487.

Dyer, S. J., O’neill, J. P., Wasel, S. M., \& Boutin, S. (2002). Quantifying barrier effects of roads and seismic lines on movements of female woodland caribou in northeastern Alberta. Canadian Journal of Zoology, 80(5), 839-845. doi:10.1139/ z02-060

Escuela de Biología. (2004). Ficha Ramsar Sitio Eco-región Lachuá. Editado por el Programa de Investigación y Monitoreo de Lachuá. Recuperado de http://studylib.es/doc/7804294/eco-regi\%C3\%B3n-lachu\%C3\%A1.

Forman, R. T. T., \& Alexander, L. E. (1998). Roads and their major ecological effects. Annual Review of Ecology and Systematics, 29(1), 207-231. doi:10.1146/annurev.ecolsys.29.1.207.

Foster, M. S., \& McDiarmid, R. W. (1983). Rhinophrynus dorsalis (Alma de Vaca, Sapo Borracho, Mexican Burrowing Toad). En D. H. Janzen (Ed.), Costa Rican Natural History (pp. 419-421). University of Chicago Press, Chicago, Illinois, United States.

Foster, W. A., Snaddon, J. L., Turner, E. C., Fayle, T. M., Cockerill, T. D., Ellwood, M. F., \& Yusah, K. M. (2011). Establishing the evidence base for maintaining biodiversity and ecosystem function in the oil palm landscapes of South East Asia. Philosophical Transactions of the Royal Society, 366(1582), 3277-3291. doi: 10.1098/ rstb.2011.0041

Frost, D. R. (2013). Amphibian Species of the World: An online reference. Version 5.6 (9 January 2013). New York, USA: American Museum of Natural History.

Gerow, K., Kline, N. C., Swann, D. E., \& Pokorny, M. (2010). Estimating annual vertebrate mortality on roads at Saguaro National Park, Arizona. $\mathrm{Hu}$ man-Wildlife Interactions, 4(2), 283-292.

Glista, D. J., DeVault, T. L., \& DeWoody, J. A. (2009). A review of mitigation measures for reducing wildlife mortality on roadways. Landscape and Urban Planning, 91(1), 1-7. doi:10.1016/j.landurbplan.2008.11.001.

Google. (2015). [Google Maps Laguna de Lachuá]. Recuperado el 3 de Junio del 2015 de https:// www.google.com/maps/place/Lachu\%C3\%A 1+Lake/@15.9487395,-90.704935,11145m/ data $=! 3 \mathrm{~m} 1$ ! $1 \mathrm{e} 3$ ! $4 \mathrm{~m} 5$ !3m4!1s0x858b038188af171b:0x6783dd6a0c335467!8m2!3d15.916075 1!4d-90.6725609

Gumier-Costa, F., \& Sperber, C. F. (2009). Atropelamentos de vertebrados na Floresta Nacional de Carajás, Pará, Brasil. Acta Amazonica, 39(2), 459-466.

Hammer, Ø, Harper, D. A. T., \& Ryan, P. D. (2001). PAST: Paleontological Statistics Software Package for Education and Data Analysis. Palaeontologia Electronica, 4(1) Art. 4. Recuperado de https://palaeo-electronica.org/2001_1/past/issue1_01.htm

Hels, T., \& Buchwald, E. (2001). The effect of road kills on amphibian populations. Biological Conservation, 99(3), 331-340. doi: 10.1016/S00063207(00)00215-9.

Hothorn, T., Brandl, R., \& Müller, J. (2012). Large-scale model-based assessment of deer-vehicle collision risk. PLoS One, 7(2), 1-10. doi: 10.1371/ journal.pone. 0029510

Instituto Nacional de Bosque [INAB]. (2003). Plan Maestro 2004-2009 del Parque Nacional Laguna Lachuá, Cobán, Alta Verapaz. Guatemala: Insti- 
tuto Nacional de Bosques, Consejo Nacional de Áreas Protegidas.

Junior, R. O., Lima, J. P., Santos, A. L. W., \& Aride, P. H. R. (2012). Caracterização da fauna de vertebrados atropelada na rodovia BR 174, Amazonas, Brasil. Revista Colombiana de Ciencia Animal, 4(2), 291-307.

Köhler, G. (2008). Reptiles of Central America. Offenbach, Frankfurt: Herpeton.

Legendre, P., \& Legendre, L. (1988). Numerical Ecology, Volume 24. Amsterdam: Elsevier.

Lesbarrères, D., Lodé, T., \& Merilä, J. (2004). What type of amphibian tunnel could reduce road kills? Oryx, 38(2), 220-223.

López, M. (2004). Los Otros Peatones. Tráfico, (julio-agosto), 36-39. Recuperado de http://www. dgt.es/revista/archivo/pdf/num167-2004-Animales.pdf

Loss, S. R., Will, T., \& Marra, P. P. (2014). Estimation of bird-vehicle collision mortality on US roads. Journal of Wildlife Management, 78(5), 763-771. doi: 10.1002/jwmg. 721

Ministerio de Agricultura, Ganadería y Alimentación [MAGA] y Centro Agronómico Tropical de Investigación y Enseñanza [CATIE]. (2001). Base de datos digital de la República de Guatemala a Escala 1: 250, 000. Coordinado por: Unidad de Políticas de e Información Estratégica (UPIE-MAGA). Guatemala.

Maxson, L. R., \& Daugherty, C. H. (1980). Evolutionary relationships of the monotypic toad family Rhinophrynidae: A biochemical perspective. Herpetologica, 36(3), 275-280.

Mazerolle, M. J. (2004). Amphibian road mortality in response to nightly variations in traffic intensity. Herpetologica, 60(1), 45-53. doi:10.1655/02109

McGarigal, K., Stafford, S., \& Cushman, S. (2000). Ordination: principal components analysis. En K. McGarigal (Ed.), Multivariate statistics for wild- life and ecology research (pp. 19-80). New York: Springer. doi:10.1007/978-1-4612-1288-1_2.

Rodríguez, A. A. (2015). Identificación y caracterización de cruces de fauna silvestre en la sección de la ampliación de la carretera nacional Ruta 32, Limón, Costa Rica (Tesis de maestría). Universidad Centro Agronómico Tropical de Investigación y Enseñanza, Costa Rica.

Rogers, O. J. (2013). Abriendo Brechas: El Proyecto Vial de la Franja Transversal del Norte, Desarrollo y Territorio en Huehuetenango. Guatemala: Centro de Estudios y Documentación de la Frontera Occidental de Guatemala (CEDFOG).

Savilaakso, S., Petrokofsky, G., Zrust, M., \& Guariguata, M. R. (2014). Palm oil and biodiversity. CIFOR Infobrief, 69(1), 1-4.

Solano, L. (2012). Contextualización histórica de la Franja Transversal del Norte (FTN). Guatemala: Centro de Estudios y Documentación de la Frontera Occidental de Guatemala.

Teixeira, F. Z., Coelho, I. P., Esperandio, I. B., Oliveira, N. R., Peter, F. P., Dornelles, S. S., ... Kindel, A. (2013). Are road-kill hotspots coincident among different vertebrate groups. Oecologia Australis, 17(1), 36-47. doi:10.4257/oeco.2013.1701.04.

Trombulak, S. C., \& Frissell, C. A. (2000). Review of ecological effects of roads on terrestrial and aquatic communities. Conservation Biology, 14(1), 18-30. doi:10.1046/j.1523-1739.2000.99084.x

van der Grift, E. A., van der Ree, R., Fahrig, L., Findlay, S., Houlahan, J., Jaeger, J. A., \& Olson, L. (2013). Evaluating the effectiveness of road mitigation measures. Biodiversity and Conservation, 22(2), 425-448. Doi:10.1007/s10531-012-0421-0.

van Perlo, B. (2006). Birds of Mexico and Central America. Princeton, Texas: Princeton University Press. 